\title{
Spotlight on cancer genomics
}

\author{
The field of cancer genomics has been advancing at a rapid pace, opening up a wealth of possibilities for \\ translational applications. In this issue, we are excited to launch a Series of commissioned articles that explore the \\ role of genomics in cancer research and oncology, from current achievements to future directions.
}

T he milestone publication of the Human Genome Project in 2001 was followed a few years later by the formation of first cancer genome cataloging projects-e.g., The Cancer Genome Atlas and the International Cancer Genome Consortium - aiming to harness stateof-the-art next-generation sequencing technologies to elucidate the alterations that underpin different cancer types. In the 12 short years since the publication of the first cancer genome (https://www.nature.com/ articles/nature07485), a surge of analytical power and genomic data has established this field as a mainstay in cancer research that is quickly emerging as an essential tool in clinical oncology. As technologies continue to improve and fundamental understanding of the cancer genome deepens, a unifying pursuit for researchers and oncologists is to leverage knowledge of genomic and other molecular features such as gene expression and epigenetic changes at the bench and at the bedside, to identify new therapeutic targets or testable biomarkers.

To highlight the ways in which the modern era of cancer genomics is changing research and clinical practice, we are pleased to introduce in this issue of Nature Cancer our Series on Clinical Cancer Genomics. The Series launches with a specially commissioned Review article by Siu and colleagues and a Cancer in Translation article by Kreisberg et al. and is accompanied by a collection of relevant primary research papers published in Nature Cancer. In the coming months, we will present additional Review articles from leaders in the field discussing the current footprint of genomics in guiding diagnosis and treatment and assisting clinical trials, and the path to bringing emerging technologies and biological discoveries to the clinic. Readers may explore the Series through a dedicated page on the Nature Cancer website that will be updated with newly published commissioned pieces and primary research articles.

The identification of discrete molecular alterations that underlie certain cancer types and the development of targeted therapies against them (such as the now-classic examples of vemurafenib for BRAF(V600E) mutations in melanoma, and tyrosine-kinase inhibitors of the BCR-ABL kinase) has propelled the implementation of molecular testing into clinical practice. With the expanding list of approved targeted therapies for 'actionable' mutations and eligible cancer types, screening patients with cancer by whole-exome sequencing and focused next-generation sequencing gene panels is becoming increasingly routine. More recently, available testing has extended to other biomarkers, such as tumor mutational burden for immune-checkpoint-blockade therapy. Currently, myriad developmental efforts to identify predictive molecular biomarkers for response to treatment, disease progression or adverse effects of therapy are underway. Such tools offer the potential to improve personalized care by identifying the most beneficial treatment for an individual, or to avoid unnecessary adverse effects or costly procedures-for example, by reducing treatments in patients likely to benefit from more-conservative therapeutic regimens.

Despite great successes, it is now clear that the precision-oncology paradigm of targeted therapy against driver alterations does not circumvent the issue of treatment-resistant and refractory disease. Many profiling studies have uncovered mechanisms of acquired or intrinsic resistance and, moreover, multi-region and single-cell sequencing have underscored spatial and subclonal heterogeneity that may impart incomplete therapeutic responses. These insights suggest that combinatorial strategies are probably necessary for effective disease management and point to the potential benefits of evaluating and targeting both the tumor and the microenvironment. Addressing therapy resistance in the clinic remains a moving target, and thus obtaining longitudinal data points across the disease and therapeutic course could facilitate earlier redirection of treatment in the event of resistance or recurrence. In the inaugural Review article of our Series, Siu and colleagues discuss the advent of 'liquid biopsy' of circulating tumor DNA in the blood, an approach that in recent years has gained traction for the detection of clinically relevant molecular biomarkers, offering opportunities for monitoring therapeutic response and recurrence with a minimally invasive approach.
With the accrual of a massive amount of data through the genomic profiling of human cancers, a central challenge remains how to systematically determine the functional importance of variants in influencing disease risk, prognosis or therapeutic potential. Collaborative consortia such as The Cancer Genome Atlas have already provided rich resources for characterizing cancer genomes and supporting translational discoveries. In 2020, the Pan-Cancer Analysis of Whole Genomes Consortium published a series of papers that characterize over 2,600 cancer whole-genome sequences with unprecedented scope and sensitivity. However, as discussed by Kreisberg et al. in a Cancer in Translation article in this issue, there remains a substantial gap between gathering molecular data at this scale and translating the findings in the clinic. Open-source databases such as CIViC are designed to make use of available genomic information by centralizing evidence and standardizing variant-annotation procedures for more-rapid clinical validation. Recognizing this limitation in previous sequencing projects, the ongoing ICGCARGO initiative will include detailed clinical annotation, to streamline translational efforts going forward.

The future role of cancer genomics in the clinic holds many exciting questions and challenges. For one, the list of actionable mutations remains far shorter than the list of those identified by molecular profiling studies. In addition to the variant-annotation efforts described above, the identification of new targetable vulnerabilities through functional genomics is a rapidly developing field. Further preclinical characterization and validation efforts may increase the clinical utility of features such as gene expression, epigenetics, immunogenomics and mutational signatures-or combinations thereof-as biomarkers or therapeutic targets. At a practical level, although costs for molecular testing are trending downward, more work is needed to ensure that next-generation sequencing and genomics-guided medicine are accessible and affordable, to benefit more patients. Moreover, it remains to be seen how emerging technologies such as singlecell RNA sequencing, with currently higher 
associated costs and sophisticated analytical requirements, may be integrated into routine clinical practice in the future.

Whether precision medicine has returned on its promise is a subject of frequent debate. Reaching this goal remains a continuous process informed by new fundamental insights into the molecular basis of cancer, increased availability of clinical data and experience, and developing technologies. With this Series and our publications looking forward, we aim to provide a nexus to showcase new clinical achievements and the foundational work to power future clinical progress. We thank our authors and referees and hope that our readers will find this selection of Nature Cancer articles enlightening and inspiring.

Published online: 20 March 2020

https://doi.org/10.1038/s43018-020-0052-4 